\title{
The Cosmic Stellar Birth and Death Rates
}

\author{
John F. Beacom \\ Department of Physics and Department of Astronomy, The Ohio State University, \\ Columbus, Ohio 43210, USA
}

\begin{abstract}
The cosmic stellar birth rate can be measured by standard astronomical techniques. It can also be probed via the cosmic stellar death rate, though until recently, this was much less precise. However, recent results based on measured supernova rates, and importantly, also on the attendant diffuse fluxes of neutrinos and gamma rays, have become competitive, and a concordant history of stellar birth and death is emerging. The neutrino flux from all past core-collapse supernovae, while faint, is realistically within reach of detection in Super-Kamiokande, and a useful limit has already been set. I will discuss predictions for this flux, the prospects for neutrino detection, the implications for understanding core-collapse supernovae, and a new limit on the contribution of type-Ia supernovae to the diffuse gamma-ray background.
\end{abstract}

Key words: cosmic star formation rate, supernovae, neutrino background, gamma-ray background

PACS: 97.60.Bw, 98.70.Vc

\section{Introduction}

The initial state that leads to a type-II (or type-Ib or type-Ic) supernova is essentially an iron white dwarf (the endpoint of nuclear fusion reactions) in the center of a massive star; when it reaches the Chandrasekhar mass, this core will collapse, along with the rest of the star. The direct microphysical messengers of the gravitational explosion are the neutrinos emitted from the hot and dense proto-neutron star. The detectable (few tens of $\mathrm{MeV}$ ) neutrinos are emitted on a timescale of several seconds, corresponding to their diffusion outward. These neutrinos from a specific type-II supernova have only been detected once, from SN 1987A (Hirata et al., 1987; Bionta et al., 1987).

The initial state that leads to a type-Ia supernova seems to be a carbonoxygen white dwarf (the endpoint of nuclear fusion reactions in a low-mass star) that accretes material from a bound binary companion; when it reaches 
the Chandrasekhar mass, it will experience runaway nuclear burning. The direct microphysical messengers of the thermonuclear explosion are the gamma rays from the nuclear decays of freshly produced ${ }^{56} \mathrm{Ni}$ and ${ }^{56} \mathrm{Co}$. The detectable (few $\mathrm{MeV}$ ) gamma rays are emitted on a timescale of up to several months, corresponding to the nuclear lifetimes. These gamma rays have never been convincingly detected from a specific type-Ia supernova, though there have been three cases in which restrictive limits were placed (e.g. Milne et al. (2004)).

The difficulty is that nearby supernovae are rare. Although gamma rays are of course easier to detect, type-Ia supernovae are more rare, and the number of emitted particles per supernova is less. In both neutrino astrophysics and gamma-ray astrophysics, the detection of a nearby supernova is a high priority. (Proposed neutrino detectors should be able to reach even to several Mpc, if neutrinos can be detected one or two at a time using a coincidence technique (Ando et al., 2005).) It should be noted immediately that the optical emission from both types of supernovae, while readily detected even at great distances, does not directly or faithfully reveal the details of the explosions or the attendant extreme conditions that allow new tests of particle physics.

I probably don't need to further convince anyone that detecting these direct messengers of supernova explosions would be good. But how? What I always say is "Everyone complains about the supernova rate, but nobody does anything about it." While we can't wish up a nearby supernova, we can attempt to detect the diffuse glows of neutrinos and gamma rays made by all past type-II and type-Ia supernovae, respectively (practically speaking, redshifts less than about $z=1$ are the most important for detection). I will argue that while this is very challenging, the signal is always there, and the prospects are quite encouraging. The parallel nature of the above discussion now develops an interesting split. The neutrino background has never been detected, but it is believed to be dominantly produced by type-II supernovae. On the other hand, the gamma-ray background has been detected, but it is now believed that the contribution from type-Ia supernovae is subdominant.

Clavton and Silk (1969) proposed long ago that the prospects for detecting the gamma-ray background from type-Ia supernovae were quite promising. It wasn't until about 15 years later that people even started considering the neutrino background from type-II supernovae; for early work, see Bisnovatvi-Kogan and Seidov (1982), Krauss. Glashow, and Schramm (1984), and Wooslev, Wilson, and Mavle (1986). Until very recently, the prospects for detecting the neutrino background from type-II supernova were thought to be at best exceedingly unlikely. However, in 2003, the Super-Kamiokande collaboration published a flux limit (Malek et al., 2003) that was about 200 times more restrictive than the earlier limit from Kamiokande (Zhang et al., 1988), and in fact in the range of realistic models. One surprising point that I will argue is that this limit on the neutrino background strongly constrains the star 

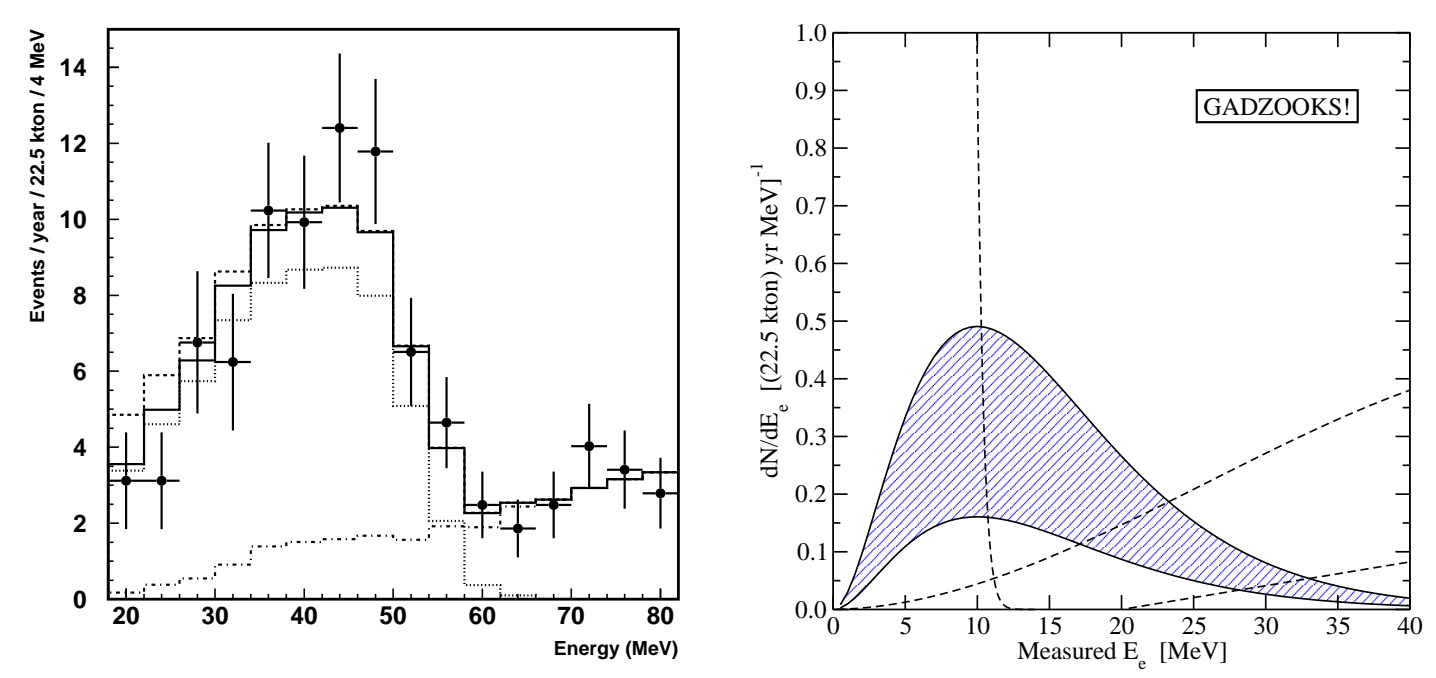

Fig. 1. Left figure: The measured spectrum (points with error bars) of $e^{-} / e^{+}$-like events in Super-Kamiokande. The solid line is the sum of the two atmospheric neutrino background components, shown as lines below the solid line. The dashed line above the solid line shows the largest allowed fit for the detector background plus the type-II supernova neutrino signal. Right figure: The projected spectrum if dissolved gadolinium is added to Super-Kamiokande. The shaded band is the allowed range of supernova neutrino signals; our more recent work (Strigari et al., 2005) favors the largest values. The atmospheric neutrino backgrounds have here been reduced by a factor $\sim 5$, which would allow a much lower threshold, more like $10 \mathrm{MeV}$, where the background from reactor neutrinos becomes overwhelming. In the figure on the right, the counts are per $1-\mathrm{MeV}$ bin, whereas on the left, they are per $4-\mathrm{MeV}$ bin. [Left figure from Malek et al. (2003). Right figure adapted from Beacom and Vagins (2004); the arXiv version defines "GADZOOKS!".]

formation rate; with reasonable assumptions, this then constrains the type-Ia supernova rate. Using this limit, or going directly from the measured type-Ia supernova rate data, one finds that the corresponding gamma-ray background is well below the measured data. Thus it now appears easier to detect the neutrino background from type-II supernovae than the gamma-ray background from type-Ia supernovae, which is certainly not what anyone expected.

I will take the Super-Kamiokande neutrino limit as a starting point, and then briefly review these constraints, following Strigari et al. (2005). The prospects for very significantly improving the Super-Kamiokande sensitivity are discussed in Beacom and Vagins (2004). In brief, it was proposed that adding about $0.2 \%$ dissolved gadolinium trichloride to Super-Kamiokande would allow the detection of neutrons by their radiative captures on gadolinium. This would then allow a coincidence detection of the positron and the neutron in $\bar{\nu}_{e}+p \rightarrow e^{+}+n$, which would greatly reduce backgrounds which can mimic the positron by itself. This proposal is undergoing extensive research and development testing by Vagins, and the prospects continue to be quite promising. 


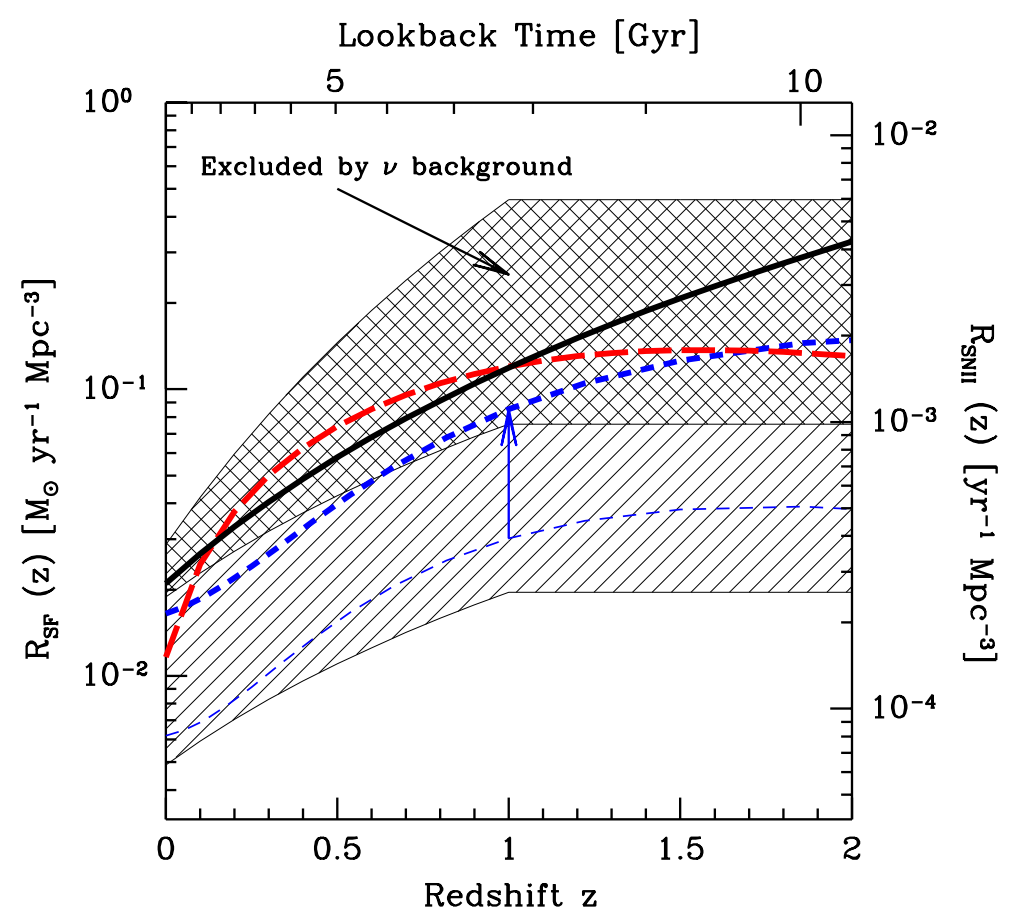

Fig. 2. Recent measurements of the cosmic star formation rate history. The entire shaded region is consistent with the results of the $2 \mathrm{dF}$ and SDSS cosmic optical spectrum. The upper cross-hatched region is ruled out by the limit on the type-II supernova neutrino flux, while the lower shaded region is allowed. Three recent (dust corrected by those authors) results are also shown in the middle of the region; in one case, the result without dust correction is shown for illustration. The heavy solid line is the result from GALEX (Schiminovich et al., 2005). The concordance region is defined by the tension between the neutrino bound and the astronomical data, and is thus concentrated at the upper edge of the lower band. [Figure taken from Strigari et al. (2005).]

\section{The Cosmic Stellar Birth and Death Rates}

Recently, the measurements of the star formation rate history have improved dramatically (e.g., see the summary in Hopkins et al. (2005)). An important aspect of this is the larger and better-understood corrections needed to compensate for obscuration by dust. As a very recent example of a new measurement, the GALEX results (Schiminovich et al., 2005) are especially noteworthy. Using the new Super-Kamiokande neutrino flux limit (Malek et al., 2003), an upper limit can be placed on the normalization of the star formation rate history. This requires making some reasonable assumptions about the neutrino emission per supernova.

In Strigari et al. (2005), we showed how the neutrino flux limit significantly 


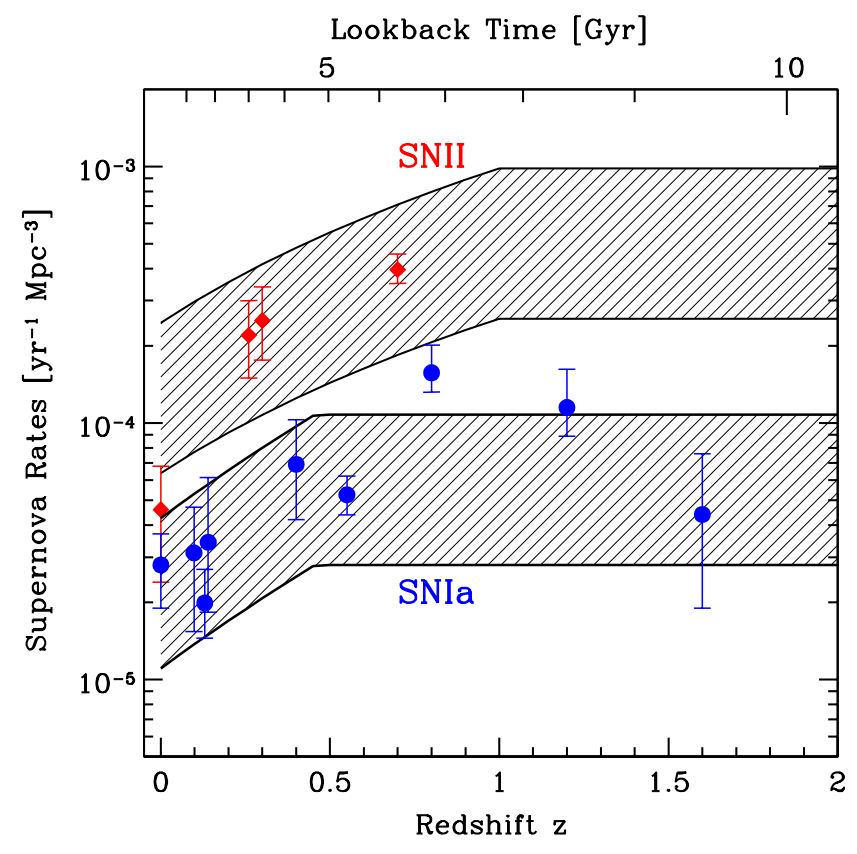

Fig. 3. The corresponding ranges for the type-II and type-Ia supernova rates, following from the allowed lower band from the previous figure. [Figure taken from Strigari et al. (2005).]

constrains the allowed range of realistic star formation rate histories. Interestingly, the dust-corrected results from GALEX, for example, come in just above our deduced limit. Very likely, this means that the neutrino emission per supernova is somewhat less than assumed. On the other hand, it also means that the true flux is probably close to the Super-Kamiokande limit, indicating that with improved sensitivity (Beacom and Vagins, 2004), a discovery could soon be made. These results for the neutrino background are similar to other recent calculations, e.g., Fukugita and Kawasaki (2003); Strigari et al. (2004); Ando and Sato (2004); Lunardini (2005); Daigne et al. (2005), up to some variations in the chosen inputs. Since the astronomical factors are already well known (and their precision is rapidly improving), a measurement of the supernova neutrino background would alternatively allow a new direct measurement of the supernova neutrino emission parameters (Yüksel et al., 2005), which would help resolve the lingering mysteries of the SN 1987A data (e.g., as displayed by Mirizzi and Raffelt (2005)).

We defined a "Concordance Model" by the tension between the upper limit from the neutrino data, and the lower limit from the astronomical data (i.e., these results with a somewhat smaller correction for dust). To validate this model, we considered the type-II and type-Ia supernova rate histories. Matching the type-II supernova rate history depends just on the assumed stellar initial mass function, whereas matching the type-Ia supernova rate history also requires making some reasonable assumptions about the formation efficiency 


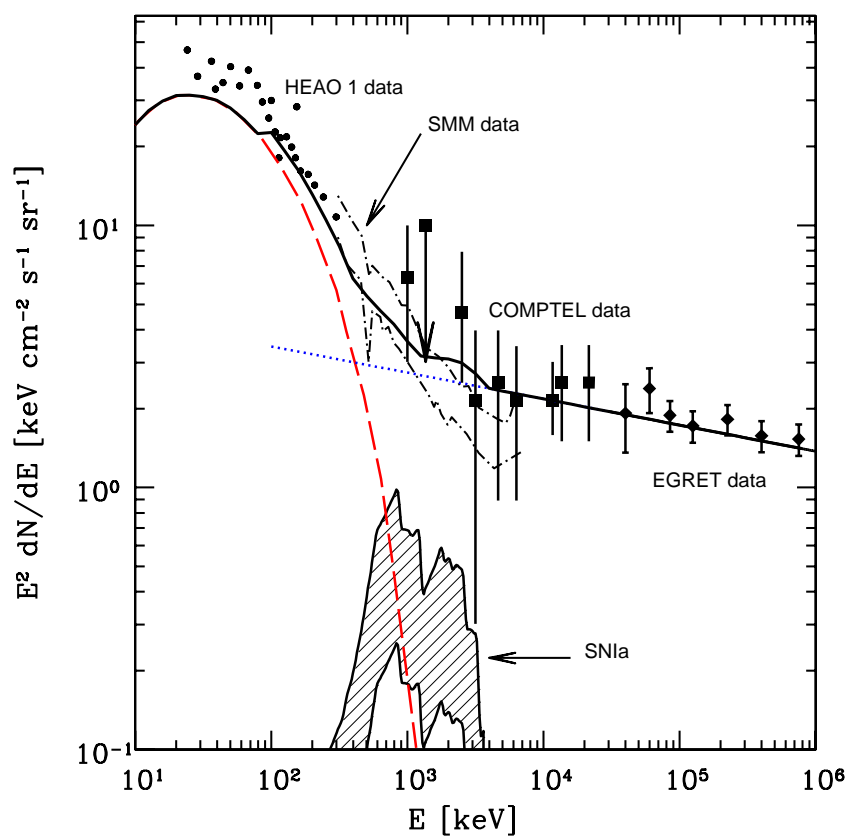

Fig. 4. Using the previous results, the prediction for the type-Ia supernova gamma-ray background; the shaded band has the same meaning as the previous figures. [Figure taken from Strigari et al. (2005).]

and typical time delay between formation and explosion (Watanabe et al., 1999). Using these results, which were also confirmed by other indicators, we predicted the gamma-ray background from type-Ia supernovae, showing that it must be far below the measured data, which is surprising (see also similar results from Ahn et al. $(2005))$.

\section{Conclusions}

Why would it be so important to detect the diffuse glows of neutrinos and gamma rays from past supernovae? That depends on your perspective, but I should begin by reminding you that, except for the $\simeq 20$ neutrinos from SN 1987A, and the at-most weak hints for the gamma rays from ${ }^{56} \mathrm{Ni} /{ }^{56} \mathrm{Co}$ from three nearby type-Ia supernovae, these emissions have never been directly detected. That's strange, and frustrating, since we know that they must be produced, and that they arise from the same physics that sources the explosions. For type-II supernovae, the neutrino emission can be directly estimated from the final mass and radius of the neutron star, which determines the gravitational energy release of $\sim 10^{53} \mathrm{erg}$. For type-Ia supernovae, the gamma ray emission can be directly estimated from the amount of synthesized ${ }^{56} \mathrm{Ni}$, which decays to ${ }^{56} \mathrm{Co}$ and then stable ${ }^{56} \mathrm{Fe}$. These decays also power the light curve, and the deduced thermonuclear energy release is $\sim 10^{51} \mathrm{erg}$. 
For the neutrino background, the existing limit is very constraining, allowing only something close to the most recent predictions for the contribution from type-II supernovae. If any other source contributed significantly, it would thus be a big surprise; the present upper limit on the flux of course applies also to any new sources. Once the neutrino flux is discovered, the spectrum shape can be tested, and the shape corresponding to type-II supernovae is quite distinctive. The primary test of new physics is thus connected with the received neutrino flux per type-II supernova. This may be affected by neutrino mixing among the known active flavors, possible mixing with postulated sterile neutrinos, neutrino decay en route, modified neutrino emission because of significant emission of new particles, etc. The neutrino emission per supernova can also be tested by effects on nucleosynthetic yields, e.g., Yoshida et al. (2005).

For the gamma-ray background, the situation is reversed, in that the observed background is a more sensitive test of new physics than the gamma ray emission per type-Ia supernova. There are measurements of the gamma-ray background, albeit with large error bars, over a broad range of energies. Just judging by the spectrum shape, there is no clear indication of the characteristic shape corresponding to the contribution from type-Ia supernovae near 1 $\mathrm{MeV}$, and there are clearly other sources at lower and higher energies. An even stronger limit on the contribution from type-Ia supernovae can be set using the star formation and type-Ia supernova rates. The properties of photons are known, and it is believed that the physical conditions of the explosion do not generally permit significant perturbations due to new physics. However, models of new physics, including the decay or annihilation of dark matter (or other particles arising in extra-dimensional models), can contribute significantly to the observed gamma-ray background. Once the measurements improve, and the astrophysical components are better understood, they can be subtracted, leading to more stringent limits on the contributions due to new physics. If the type-Ia supernova contribution can somehow be isolated (Zhang and Beacom, 2004), it will provide a new test of the gamma-ray emission per supernova and the evolution of the supernova rate, both of which could help improve the understanding required for using type-Ia supernovae as standard candles to measure dark energy.

To summarize, a better understanding of the diffuse neutrino and gamma-ray backgrounds, and specifically the portions associated with supernovae, would have important implications for several fields:

- Particle physics: Neutrino properties; novel energy-loss channels in typeII supernovae; etc.

- Nuclear physics: Production of the light and heavy elements; neutron star equation of state; etc.

- Astrophysics: Cycle of stellar birth, life, and death; constraints on new sources; etc. 
- Cosmology: Supernova distance indicators and dark energy; dark matter decay or annihilation; etc.

Thus the faint fossil records of supernovae, revealed by their diffuse neutrino and gamma ray backgrounds, offer a challenging but very promising route to better understanding the physics of their explosions.

This work was supported by The Ohio State University. I thank the organizers for the invitation to this very nice meeting, and also especially Eli Dwek, Peter Höflich, and Stan Woosley for their interesting and helpful questions and comments on my talk. I thank Shin'ichiro Ando, Louie Strigari, Mark Vagins, Terry Walker, Hasan Yüksel, and Pengjie Zhang for very enjoyable collaborations on this and closely related work.

\section{References}

K. Ahn, E. Komatsu and P. Höflich, "Cosmic gamma-ray background from Type Ia supernovae revisited: Evidence for missing gamma-rays at MeV," Phys. Rev. D 71, 121301 (2005) arXiv:astro-ph/0506126.

S. Ando, J. F. Beacom and H. Yüksel, "Detection of neutrinos from supernovae in nearby galaxies," Phys. Rev. Lett. 95, 171101 (2005) arXiv:astro-ph/0503321.

S. Ando and K. Sato, "Relic neutrino background from cosmological supernovae," New J. Phys. 6, 170 (2004) arXiv:astro-ph/0410061.

J. F. Beacom and M. R. Vagins, "Antineutrino spectroscopy with large water Cherenkov detectors," Phys. Rev. Lett. 93, 171101 (2004) arXiv:hep-ph/0309300.

R. M. Bionta et al. [IMB Collaboration], "Observation of a neutrino burst in coincidence with Supernova 1987A In the Large Magellanic Cloud," Phys. Rev. Lett. 58, 1494 (1987).

G. S. Bisnovatyi-Kogan and Z. F. Seidov, "Medium-energy neutrinos in the universe," Sov. Astron. 26, 132 (1982).

D. D. Clayton and J. Silk, "Measuring the rate of nucleosynthesis with a gamma-ray detector," Astrophys. J. 158, L43 (1969).

F. Daigne, K. A. Olive, P. Sandick and E. Vangioni, "Neutrino signatures from the first stars," arXiv:astro-ph/0509404.

M. Fukugita and M. Kawasaki, "Constraints on the star formation rate from supernova relic neutrino observations," Mon. Not. Roy. Astron. Soc. 340, L7 (2003) arXiv:astro-ph/0204376.

K. Hirata et al. [Kamiokande Collaboration], "Observation of a neutrino burst from Supernova 1987A," Phys. Rev. Lett. 58, 1490 (1987).

A. M. Hopkins, S. M. Rao and D. A. Turnshek, "The star formation history of damped Lyman alpha absorbers," Astrophys. J. 630, 108 (2005) arXiv:astro-ph/0505418. 
L. M. Krauss, S. L. Glashow and D. N. Schramm, "Antineutrino astronomy and geophysics," Nature 310, 191 (1984).

C. Lunardini, "The diffuse supernova neutrino flux, star formation rate and SN1987A," arXiv:astro-ph/0509233.

M. Malek et al. [Super-Kamiokande Collaboration], "Search for supernova relic neutrinos at Super-Kamiokande," Phys. Rev. Lett. 90, 061101 (2003) arXiv:hep-ex/0209028.

P. A. Milne et al., "Unified 1-D simulations of gamma-ray line emission from type Ia supernovae," Astrophys. J. 613, 1101 (2004) arXiv:astro-ph/0406173.

A. Mirizzi and G. G. Raffelt, "New analysis of the SN 1987A neutrinos with a flexible spectral shape," Phys. Rev. D 72, 063001 (2005) arXiv:astro-ph/0508612.

D. Schiminovich et al. [The GALEX-VVDS Collaboration], "The GALEXVVDS measurement of the evolution of the far-ultraviolet luminosity density and the cosmic star formation rate," Astrophys. J. 619, L47 (2005) arXiv:astro-ph/0411424.

L. E. Strigari, M. Kaplinghat, G. Steigman and T. P. Walker, "The supernova relic neutrino backgrounds at KamLAND and Super-Kamiokande," J. Cosmol. Astropart. Phys. 0403, 007 (2004) arXiv:astro-ph/0312346.

L. E. Strigari, J. F. Beacom, T. P. Walker and P. Zhang, "The concordance cosmic star formation rate: Implications from and for the supernova neutrino and gamma ray backgrounds," J. Cosmol. Astropart. Phys. 0504, 017 (2005) arXiv:astro-ph/0502150.

K. Watanabe, D. H. Hartmann, M. D. Leising and L. S. The, "The diffuse gamma-ray background from supernovae," Astrophys. J. 516, 285 (1999) arXiv:astro-ph/9809197.

S. E. Woosley, J. .R. Wilson, and R. Mayle, "Gravitational collapse and the cosmic antineutrino background," Astrophys. J. 302, 19 (1986).

P. J. Zhang and J. F. Beacom, "Angular correlations of the $\mathrm{MeV}$ cosmic gamma ray background," Astrophys. J. 614, 37 (2004) arXiv:astro-ph/0401351.

W. Zhang et al. [Kamiokande Collaboration], "Experimental limit on the flux of relic anti-neutrinos from past supernovae," Phys. Rev. Lett. 61, 385 (1988).

T. Yoshida, T. Kajino and D. H. Hartmann, "Constraining the spectrum of supernova neutrinos from nu-process-induced light-element synthesis," Phys. Rev. Lett. 94, 231101 (2005) arXiv:astro-ph/0505043.

H. Yüksel, S. Ando and J. F. Beacom, "Direct measurement of supernova neutrino emission parameters with a gadolinium enhanced Super-Kamiokande detector," arXiv:astro-ph/0509297. 